\title{
A Commentary on the Application of Artificial Intelligence in the Insurance Industry
}

\author{
Sushant $K$ Singh $^{1^{*}(\mathbb{D})}$ and Muralidhar Chivukula ${ }^{2}$ \\ ${ }^{1}$ Artificial Intelligence and Analytics / Health Care and Life Sciences, Virtusa Corporation, New York, NY, USA \\ ${ }^{2}$ Data Management \& Analytics / Insurance, Virtusa Corporation, New York, NY, USA
}

\begin{abstract}
Millions of uninsured individuals in the US live in the areas, which are highly vulnerable to health and other risks. Artificial intelligence (AI) has become one-point solution for a variety of socioeconomic, health, environmental, technological, and business challenges and insurance industry is not an exception. This article highlights how machine learning (ML) and deep learning (DL), the two pillars of Al, help to resolve a variety of challenges in the insurance industry. The data produced in the insurance industry is unique, and the challenges are complex. Therefore, solutions have to be designed accordingly. The application of Al in the insurance industry is still in the rudimentary stages. Traditional statistical and ML methods may not do justice while developing various prediction models. Data scientists and engineers have to work together and get their hands dirty to find innovative ways of resolving them as well as developing robust and sustainable solutions that could last for years. These professionals require a very strong research background in handling a variety of data and abstract thinking style with an analytical brain. The daunting goals cannot be accomplished in a typical lift-andshift approach. Reinforcement and transfer learnings, ensemble models, natural language understanding, processing, and generation, and DL could help. Innovations in the insurance industry and emerging technologies such as Drone, the Internet of Things, and Fitbit would be brought additional challenges to Al professionals.
\end{abstract}

\section{Keywords}

Insurance, Artificial Intelligence, Machine Learning, Deep Learning

\section{Introduction}

Insurance is one of the principal industries in the US, covers nearly 3\% (\$507 billion) of its GDP, and collects approximately $\$ 1$ trillion in premiums each year [1]. On the other hand, insurance fraud has become a monstrous challenge as it costs over $\$ 40$ billion per year that triggers a premium increase of between $\$ 400$ and $\$ 700$ per year for the US families [1]. A majority of the insurance fraud is non-health in nature. According to the Federal Bureau of Investigation (FBI) of the US, premium diversion, fee churning, asset diversion, and workers' compensation fraud are some of the common fraudulent schemes [1]. Also, disaster-related frauds are massive and very difficult to identify. Only in the US in $2005 \$ 6$ billion possibly was a fraudulent amount claimed due to the Hurricane Caterina [1]. Globally, nearly $\$ 477$ billion is lost between 1970 and 2017 due to the catastrophic events [2]. It is imperative that 11 US states have more than $10 \%$ uninsured population who are also vulnerable to various health challenges including but not limited to smoking, obesity, physical inactivity, low birth rate, drug deaths, environmental pollutions, and lack of doctors [3], and a variety of risks including personal and residential, financial, road, workplace, and emergency preparedness safety risks [4] (Figure 1).
The irony is that a majority of uninsured individuals live in the highest health vulnerable areas (Figure 1). In addition to the fraudulent activities, the insurance industry encounters several fundamental challenges on a daily basis such as efficient handling of underwritings. However, modification in regulations and standards, change in company focus, the speed of technological change, varying customer behaviors and expectations, and competition from new market entrants are perceived as the daunting challenges by a majority of insurance leaders [5]. The survival strategies of most of the insurance companies are to adopting new core systems, automation of all the processes, and the application of Artificial Intelligence (Al) [5]. In this perspective article, we are focusing on how Al can help in identifying some critical challenges and

*Corresponding author: Sushant K Singh, Artificial Intelligence \& Analytics | Health Care \& Life Sciences, Virtusa Corporation, New York, NY, USA

Accepted: February 18, 2020

Published online: February 20, 2020

Citation: Singh SK, Chivukula M (2020) A Commentary on the Application of Artificial Intelligence in the Insurance Industry. Trends Artif Intell 4(1):75-79 


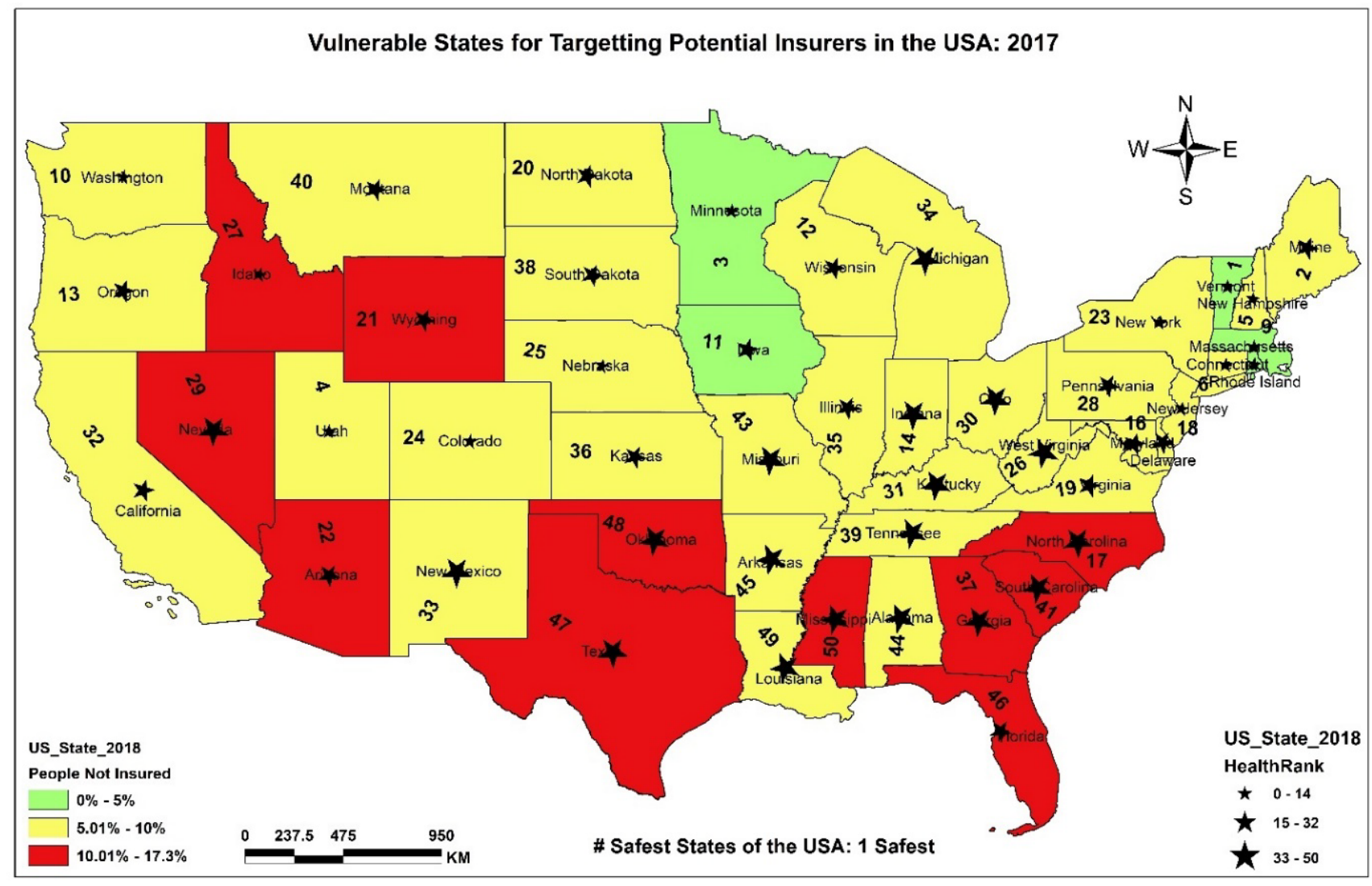

Figure 1: The Vulnerable US States to target potential insurers ${ }^{\mathrm{a}}$.

aThe map was created for this perspective article using ArcGIS version 10.6 for the Windows environment.

provide possible solutions in the insurance industry.

The $\mathrm{Al}$ and it's applications have emerged over thousands of year date back to $300 \mathrm{BC}$, however, in the modern era, we define it as a science of training a machine to perform a specific function that a human brain cannot perform when the information is in bulk and requires complex processes. Machine learning $(\mathrm{ML})$ and deep learning (DL) are the two pillars of Al through which all the Al goals can be achieved.

\section{Artificial Intelligence and the Insurance In- dustry}

\section{Automating underwriting}

Underwriting is one of the biggest challenges in the insurance industry and other industries as well because it heavily depends on human interventions. Therefore, the likelihood of human errors is very high. An automated underwriting expert system has been developed as early as in late 19s in Canada [6]. Later, other ML and statistical techniques such as fuzzy logic and natural language processing (NLP) have been applied to accomplish underwriter automation [7].

\section{Prospecting clients}

Al can be used in prospecting clients for a variety of insurance policies including but not limited to life, health, annuities, disability, liabilities, auto, homeowner, renters, flood, earthquake, wildfire, pet, hail, agriculture, and reinsurance. Chi-square Automatic Interaction Detector (CHAID), Support Vector Machines (SVM), Hierarchical Clustering (HC), k-Nearest Neighbor (k-NN), and Lasso have been applied in developing predictive models in prospecting clients [8]. The task is not so simple; it encounters several challenges such as imbalanced and missing data. However, Al can efficiently mitigate them by applying appropriate imputation and sampling techniques.

\section{Targeting new business}

Targeting new business plays a vital role in the success of any businesses, though it is very challenging. Geospatial analytics can help in efficiently locating a new business where zip code level data on the demographic, socioeconomic, health, environmental, and other relevant information help the decision makers to identify potential new business [9].

\section{Personalized services}

Personalized services are in high demand from the customers as it helps match their needs and lifestyle. SVM and the latent class model have helped in developing prediction models for personalized services using customers' product ratings [10] or their demographic data, preferences, interaction, behavior, attitude, lifestyle details, interests, and hobbies. 


\section{Customer segmentation}

Each is unique and has varied behavior and attitude that impact their shopping behaviors and responses to the products they are offered. Customer segmentation helps significantly in understanding a diverse customer group. Multinomial regression and count regression models have been used in customer segmentation in insurance where customers' socioeconomic, demographic, and their frequency of various channels of communication were used [11]. Fuzzy analytic network process-based weighted recency, frequency, monetary value model and $\mathrm{K}$-means have helped an auto insurance company in efficiently segmenting their customers into 'new,' 'risky,' 'uncertain,' and 'best' [12]. Customer segmentation also helped in understanding the insurance consumption across various communication channels [13]. Hybrid models could be more efficient and accurate in customer segmentation where the strengths of clustering, classification, and rule mining algorithms can be applied together [14]. Other algorithms have helped in efficiently segmenting the customers not listed here.

\section{Customer churn prediction}

A variety of $M L$ algorithms have been used to predict whether a customer would churn or not across many industries including insurance [15]. Logistic regression, decision trees, SVN, artificial neural network (ANN), and several other classification algorithms have helped efficiently predicting customer churn [15]. Considering the complexity of customers' data, hybrid models such as the logit leaf model could be advantageous over other traditional algorithms [16]. Adding term frequency and inverse document frequency to base classifiers such as SVM, Naïve Bayes, and J4.8 have significantly increased the prediction accuracy in customer churn prediction [17].

\section{Customer lifetime value prediction}

From the companies' perspective, customers lifetime value (CLV) is vital in understanding customers' financial value to a company. Various ML algorithms such as classification and regression trees (CART), SVM, additive regression, K-Star Method, multilayer perceptron, wavelet neural network [18], probit, regression, and Naïve use customers' purchased products or services, socioeconomic, and demographic information to predict the CLV that helps understand the probability of the customers' attitude and behavior towards successfully maintaining their policies [19]. Other advanced approaches such as synthetic minority oversampling combined with deep neural networks [20] have shown some promising results in CLV prediction.

\section{Fraud prediction}

Insurance fraud steals a huge amount of money from the insurance companies' profits every year. A variety of $\mathrm{ML}$ and $\mathrm{DL}$ algorithms have been used in fraud detection including but not limited to RF-based multiple classifier systems, PCA, k-NN [21], RF, SVM, however, hybrid models have shown some promising results such as deep neural network and Latent Dirichlet allocation (LDA) [22]. It is a known fact that fraud prediction requires enough and constant flow of data as the fraud patterns change quickly. Therefore, reinforcement and cognitive learning's and appropriate use of NLP would help develop real-time and most accurate fraud prediction models.

\section{Loss prediction}

Insurance or premium loss is another challenge in the insurance industry. Al has also helped in rescuing the industry by predicting loss using a variety of $\mathrm{ML}$ algorithms including but not limited to Bayesian multivariate and non-linear hierarchical models, gradient boosting and generalized linear models [23].

\section{Claim rate prediction}

With new entrants, the insurance industry has become extremely competitive. Al helps them to be one step ahead of their competitors through forecasting the upcoming claims that help the companies to charge competitive premiums to the customers.

\section{Life event marketing}

Al also helps in targeting the right person at a right time so that the customer can be approached with the right message. Automated marketing is key to revealing the insights of the customers' attitude and behavior via initial research, product inquiry, purchases, and claims. Al could also help to extract the special life events data from multiple communication channels and target them for appropriate marketing.

\section{Recommendation engine}

A customer who needs health insurance would be expecting offers on health insurance, not auto insurance. This is where recommendation engines play a crucial role to recommend appropriate offers for each specific client. Customers' sentiments and their preferences can be used in developing personalized recommendation [24].

\section{Insurance chatbot}

Chatbots are one of the revolutionary inventions of $\mathrm{Al}$ that help millions of customers to answer their basic questions and concerns through online conversations or messaging [25].

Data generated in the insurance industry is different and complex when compared with other industries where financial transactions occur. Therefore, the challenges are unique and complex that require customized solutions. Moreover, recent innovations in the insurance industry have brought critical challenges to data scientists. For example, data on digital channel communication and interactions, customer experience, managing product life-cycle, designing and configuring new products, and preventing loss would require customized methodological approaches. Additionally, emerging technologies including Drone and loT devices such as fitness, health, driving patterns, user compliance, and risk indicators produce enormous data that would help in developing customized insurance products, creating appropriate marketing and sales 
strategies, personalized customer services, insurance claims and fraud at the same time it would be challenging for data scientists and engineers as they may not have encountered these challenges before. In a recent study, the authors have used a variety of ML algorithms to predict individuals' awareness based on their socioeconomic, demographic, psychological, and socio-behavioral data. They highlight that traditional statistical or ML methods may not be effective in such cases as the problem could be a non-linear classification as opposed to linear classification [26].

By integrating geolocation data, data from public sources such as the Census, social network sites, customer-shared information, data produced from the IoT, data acquired from the third party could be used in several challenges such as efficiently automating underwriter using Al techniques [27]. A variety of Al techniques such as chatbots, digital personal assistants, robo-advisors, cognitive computing, NLP, ML, DL, autonomous things, robotic process automation would assist at a greater extent, however, if not properly applied it could bounce back. Therefore, traditional statistical and machine learning approaches may not be efficient, thus applied data science methods are warranted. For example, image processing, text analytics, NLP, NLU, and NLG can help in product development, marketing, and risk ratings after extracting data from millions of policy and legal documents. Speech recognition techniques would help in understanding customers' challenges and improvise the existing and future services.

Similarly, video analysis integrated with image processing and facial recognition techniques would help in efficiently understand customers' emotions and personalized needs [27]. Al can also help in analyze and predict products buying behaviors, subscription or churn behavior prediction; customer-level marketing strategies (right product at right time) prediction, potential and leads customer prediction, zip-code level products and services prediction, analyzing and predicting customers hotspots, customers' buying behaviors; customer-centric campaign strategies predictions; customers' sentiment analysis and prediction; real-time spatial decision support system for pre, ongoing, and post-campaign activities [27]. Therefore, Al can certainly help to resolve the existing and upcoming challenges if they are clearly understood, and the solutions are appropriately designed. Additionally, Al would bring an array of business benefits to the insurance industry including but not limited to customer satisfaction and retention, interaction improvement, sales and service completion, lower cost of servicing and sales, improved customer intelligence, contextualization and personalization of content, efficiency, and lower cost of operations. Data science and engineering professionals have to collaborate and help the insurance industry to survive and serve society as well sustainably. Al could be a double-edged sword, so it has to be applied accordingly.

\section{Acknowledgment}

The authors acknowledge all data scientists and engineers for their hard work. The authors did not receive any grant for this study; declares no conflict of interests, and the interpretations presented here are purely for research purposes and do not necessarily represent the official views of the organization authors are associated with.

\section{References}

1. https://www.fbi.gov/stats-services/publications/insurance-fraud

2. https://www.statista.com/statistics/267210/natural-disaster-damage-totals-worldwide-since-1970/

3. (2017) America's health rankings: A call to action for individuals and their communities USA. United Health Foundation (UHF).

4. McCann A (2018) 2018's Safest States in America. Wallethub, USA.

5. PwC (2018) Insurance Top Issues. PwC Finacial Services, USA.

6. Pilote M, M Fillion (1991) Automated underwriting at Continental Canada: Results and major obstacles. Artificial Intelligence Applications on Wall Street, 1991. Proceedings. First International Conference on, IEEE.

7. Aggour KS, PP Bonissone, WE Cheetham, et al. (2006) Automating the underwriting of insurance applications. Al Magazine 27-36.

8. Simester D, A Timoshenko, SI Zoumpoulis (2019) Targeting prospective customers: Robustness of machine learning methods to typical data challenges. Management Science.

9. Singh SK (2016) Geospatial analysis of census data for targeting new businesses using geoeconomics. Journal of Intelligence Studies in Business 6: 5-12.

10. Cheung KW, JT Kwok, MH Law, et al. (2003) Mining customer product ratings for personalized marketing. Decision Support Systems 35: 231-243.

11. Dalla Pozza I, A Brochado, L Texier, et al. (2018) Multichannel segmentation in the after-sales stage in the insurance industry. International Journal of Bank Marketing 36: 1055-1072.

12. Ravasan AZ, T Mansouri (2018) A fuzzy ANP based weighted RFM model for customer segmentation in auto insurance sector. Intelligent Systems: Concepts, Methodologies, Tools, and Applications, IGI Global 1050-1067.

13. Mau S, IP Cvijikj, J Wagner (2018) Understanding the differences in customer portfolio characteristics and insurance consumption across distribution channels. University of Lausanne, 56-58.

14. Khalili-Damghani K, F Abdi, S Abolmakarem (2018) Hybrid soft computing approach based on clustering, rule mining, and decision tree analysis for customer segmentation problem: Real case of customer-centric industries. Applied Soft Computing 73: 816-828.

15. Vafeiadis T, KI Diamantaras, G Sarigiannidis, et al. (2015) A comparison of machine learning techniques for customer churn prediction. Simulation Modelling Practice and Theory 55: 1-9.

16. De Caigny A, K Coussement, KW De Bock (2018) A new hybrid classification algorithm for customer churn prediction based on logistic regression and decision trees. European Journal of Operational Research 269: 760-772.

17. Morik K, H Köpcke (2004) Analysing customer churn in insurance data - A case study. European Conference on Principles of Data Mining and Knowledge Discovery 3202: 325-336

18. Rathi T, V Ravi (2017) Customer lifetime value measurement using machine learning techniques. Artificial Intelligence: Con- 
cepts, Methodologies, Tools, and Applications, IGI Global: 30133022.

19. Verhoef PC, B Donkers (2001) Predicting customer potential value an application in the insurance industry. Decision Support Systems 32: 189-199.

20. Sifa R, J Runge, C Bauckhage, et al. (2018) Customer lifetime value prediction in non-contractual freemium settings: Chasing high-value users using deep neural networks and SMOTE. Proceedings of the 51st Hawaii International Conference on System Sciences 923-932.

21. Li Y, C Yan, W Liu, et al. (2018) A principle component analysis-based random forest with the potential nearest neighbor method for automobile insurance fraud identification. Applied Soft Computing 70: 1000-1009.

22. Wang Y, W Xu (2018) Leveraging deep learning with LDA-based text analytics to detect automobile insurance fraud. Decision Support Systems 105: 87-95.
23. Zhang Y, V Dukic (2013) Predicting multivariate insurance loss payments under the bayesian copula framework. Journal of Risk and Insurance 80: 891-919.

24. Jing N, T Jiang, J Du, et al. (2018) Personalized recommendation based on customer preference mining and sentiment assessment from a Chinese e-commerce website. Electronic Commerce Research 18: 159-179.

25. Riikkinen M, H Saarijärvi, P Sarlin, et al. (2018) Using artificial intelligence to create value in insurance. International Journal of Bank Marketing 36: 1145-1168.

26. Singh SK, RW Taylor, MM Rahman, et al. (2018) Developing robust arsenic awareness prediction models using machine learning algorithms. Journal of Environmental Management 211: 125137.

27. Bratteli AK (2018) Transforming insurance in an era of disruption insight. Gartner, USA.

DOI: $10.36959 / 643 / 305$

Copyright: (c) 2020 Singh et al. This is an open-access article distributed under the terms of the Creative Commons Attribution License, which permits unrestricted use, distribution, and reproduction in any medium, provided the original author and source are credited. 